\title{
Three-coloring triangle-free planar graphs in linear time*
}

\author{
Zdeněk Dvořák ${ }^{\dagger} \quad$ Ken-ichi Kawarabayashi \\ Robin Thomas ${ }^{\S}$
}

1 April 2009

\begin{abstract}
Grötzsch's theorem states that every triangle-free planar graph is 3-colorable, and several relatively simple proofs of this fact were provided by Thomassen and other authors. It is easy to convert these proofs into quadratic-time algorithms to find a 3-coloring, but it is not clear how to find such a coloring in linear time (Kowalik used a nontrivial data structure to construct an $O(n \log n)$ algorithm).

We design a linear-time algorithm to find a 3-coloring of a given triangle-free planar graph. The algorithm avoids using any complex data structures, which makes it easy to implement. As a by-product we give a yet simpler proof of Grötzsch's theorem.
\end{abstract}

\section{Introduction}

The following is a classical theorem of Grötzsch [6].

\footnotetext{
*An extended abstract of this paper appeared in ACM-SIAM Symposium on Discrete Algorithms (SODA'09). Published in ACM Transactions on Algorithms 7 (2011), Article 41, 14 pages.

${ }^{\dagger}$ Department of Applied Mathematics, Charles University, Prague, Czech Republic.

${ }^{\ddagger}$ National Institute of Informatics, 2-1-2 Hitotsubashi, Chiyoda-ku, Tokyo 101-8430, Japan.

§School of Mathematics, Georgia Institute of Technology, Atlanta, Georgia 30332-0160, USA. Partially supported by NSF under Grant No. DMS-0701077.
} 
Theorem 1.1. Every triangle-free planar graph is 3-colorable.

This result has been the subject of extensive research. Thomassen [15, 16] found two short proofs and extended the result in many ways. We return to the various extensions later, but let us discuss algorithmic aspects of Theorem 1.1 first. It is easy to convert either of Thomassen's proofs into a quadratic-time algorithm to find a 3-coloring, but it is not clear how to do so in linear time. A serious problem appears very early in the algorithm. Given a facial cycle $C$ of length four, one would like to identify a pair of diagonally opposite vertices of $C$ and apply recursion to the smaller graph. It is easy to see that at least one pair of diagonally opposite vertices on $C$ can be identified without creating a triangle, but how can we efficiently decide which pair? If we could test in (amortized) constant time whether given two vertices are joined by a path of length at most three, then that would take care of this issue. This can, in fact, be done, using a data structure of Kowalik and Kurowski [8] provided the graph does not change. In our application, however, we need to repeatedly identify vertices, and it is not clear how to maintain the data structure of Kowalik and Kurowski in overall linear time. Kowalik [7] developed a sophisticated enhancement of this data structure that supports edge addition and deletion in amortized $O(\log n)$ time. Furthermore, he found a variant of the proof of Grötzsch's theorem that can be turned into an $O(n \log n)$ algorithm to 3-color a triangle-free planar graph on $n$ vertices using this data structure. We improve this to a linear-time algorithm, as follows.

Theorem 1.2. There is a linear-time algorithm to 3-color an input trianglefree planar graph.

To describe the algorithm we exhibit a specific list of five reducible configurations, called "multigrams", and show that every triangle-free planar graph contains one of those reducible configurations. Proving this is the only step that requires some effort; the rest of the algorithm is entirely straightforward, and the algorithm is very easy to implement. Given a triangle-free planar graph $G$ we look for one of the reducible configurations in $G$, and upon finding one we modify $G$ to a smaller graph $G^{\prime}$, and apply the algorithm recursively to $G^{\prime}$. It is easy to see that every 3 -coloring of $G^{\prime}$ can be converted to a 3-coloring of $G$ in constant time. Furthermore, each reducible configuration has a vertex of degree at most three, and, conversely, given a vertex of $G$ of degree at most three it can be checked in constant time whether it belongs to 
a reducible configuration. Thus at every step a reducible configuration can be found in amortized constant time by maintaining a list of candidates for such vertices. As a by-product of the proof of correctness of our algorithm we give a short proof of Grötzsch's theorem.

Let us briefly survey some of the related work. Since in a proof of Theorem 1.1 it is easy to eliminate faces of length four, the heart of the argument lies in proving the theorem for graphs of girth at least five. For such graphs there are several extensions of the theorem. Thomassen proved in [15] that every graph of girth at least five that admits an embedding in the projective plane or the torus is 3-colorable, and the analogous result for Klein bottle graphs was obtained in [14]. For a general surface $\Sigma$, Thomassen [17] proved the deep theorem that there are only finitely many 4-critical graphs of girth at least five that embed in $\Sigma$. (A graph is 4-critical if it is not 3-colorable, but every proper subgraph is.)

None of the results mentioned in the previous paragraph hold without the additional restriction on girth. Nevertheless, Gimbel and Thomassen [5] found an elegant characterization of 3-colorability of triangle-free projectiveplanar graphs. That result does not seem to extend to other surfaces, but two of us in a joint work with Král' [3] were able to find a sufficient condition for 3-colorability of triangle-free graphs drawn on a fixed surface $\Sigma$. The condition is closely related to the sufficient condition for the existence of disjoint connecting trees in [12]. Using that condition Dvořák, Král' and Thomas were able to design a linear-time algorithm to test if a triangle-free graph on a fixed surface is 3-colorable [3].

If we allow the planar graph $G$ to have triangles, then testing 3-colorability becomes NP-hard [4]. There is an interesting conjecture of Steinberg stating that every planar graph with no cycles of length four or five is 3-colorable, but that is still open. Every planar graph is 4-colorable by the Four-Color Theorem [1, 2, 11], and a 4-coloring can be found in quadratic time [11]. Any improvement to the running time of this algorithm would seem to require new ideas. A 5-coloring of a planar graph can be found in linear time [10].

Our terminology is standard. All graphs in this paper are simple and paths and cycles have no repeated vertices. By a plane graph we mean a graph that is drawn in the plane. On several occasions we will be identifying vertices, but when we do, we will remove the resulting parallel edges. When this will be done by the algorithm we will make sure that the only parallel edges that arise will form faces of length two. The detection and removal of such parallel edges can be done in constant time. 


\section{Short proof of Grötzsch's theorem}

Let $G$ be a plane graph. Somewhat nonstandardly, we call a cycle $F$ in $G$ facial if it bounds a face in a connected component of $G$, regardless of whether $F$ is a face or not (another component of $G$ might lie in the disk bounded by $F$ ). This technicality makes no difference in this section, because here we may assume that all graphs are connected. However, it will be needed in the description of the algorithm, because the graph may become disconnected during the course of the algorithm, and we cannot afford to decompose it into connected components.

By a tetragram in $G$ we mean a sequence $\left(v_{1}, v_{2}, v_{3}, v_{4}\right)$ of vertices of $G$ such that they form a facial cycle in $G$ in the order listed. We define a hexagram $\left(v_{1}, v_{2}, \ldots, v_{6}\right)$ similarly. By a pentagram in $G$ we mean a sequence $\left(v_{1}, v_{2}, v_{3}, v_{4}, v_{5}\right)$ of vertices of $G$ such that they form a facial cycle in $G$ in the order listed and $v_{1}, v_{2}, v_{3}, v_{4}$ all have degree exactly three. We will show that every triangle-free planar graph of minimum degree at least three has a tetra-, penta- or hexagram with certain additional properties that will allow an inductive argument. But first we need the following lemma.

Lemma 2.1. Let $G$ be a connected triangle-free plane graph and let $f_{0}$ be the unbounded face of $G$. Assume that the boundary of $f_{0}$ is a cycle $C$ of length at most six, and that every vertex of $G$ not on $C$ has degree at least three. If $G \neq C$, then $G$ has either a tetragram, or a pentagram $\left(v_{1}, v_{2}, v_{3}, v_{4}, v_{5}\right)$ such that $v_{1}, v_{2}, v_{3}, v_{4} \notin V(C)$.

Proof. We define the charge of a vertex $v$ to be $3 \operatorname{deg}(v)-12$, the charge of the face $f_{0}$ to be $3|V(C)|+11$ and the charge of a face $f \neq f_{0}$ of length $\ell$ to be $3 \ell-12$. It follows from Euler's formula that the sum of the charges of all vertices and faces is -1 .

We now redistribute the charges according to the following rules. Every vertex not on $C$ of degree three will receive one unit of charge from each incident face, each vertex on $C$ of degree three will receive three units from $f_{0}$, and each vertex of degree two on $C$ will receive five units from $f_{0}$ and one unit from the other incident face. Thus the final charge of every vertex is non-negative.

We now show that the final charge of $f_{0}$ is also non-negative. Let $\ell$ denote the length of $C$. Then $f_{0}$ has initial charge of $3 \ell+11$. By hypothesis at least one vertex of $C$ has degree at least three, and hence $f_{0}$ sends a total of at 
most $5(\ell-1)+3$ units of charge, leaving it at the end with charge of at least $3 \ell+11-5(\ell-1)-3 \geq 1$.

Since no charge is lost or created, there is a face $f \neq f_{0}$ whose final charge is negative. Since $f$ sends at most one unit to each incident vertex, we see that $f$ has length at most five. Furthermore, if $f$ has length exactly five, then it sends one unit to at least four incident vertices. None of those could be a degree two vertex on $C$, for then $f$ would not be sending anything to the ends of the common subpath of the boundaries of $f$ and $f_{0}$. Thus the vertices of $f$ form the desired tetragram or pentagram.

Let $k=4,5,6$, and let $\left(v_{1}, v_{2}, \ldots, v_{k}\right)$ be a tetragram, pentagram or hexagram in a triangle-free plane graph $G$. If $k=4$ or $k=6$, then we say that $\left(v_{1}, v_{2}, \ldots, v_{k}\right)$ is safe if every path in $G$ of length at most three with ends $v_{1}$ and $v_{3}$ is a subgraph of the cycle $v_{1} v_{2} \cdots v_{k}$. For $k=5$ we define safety as follows. For $i=1,2,3,4$ let $x_{i}$ be the neighbor of $v_{i}$ distinct from $v_{i-1}$ and $v_{i+1}$ (where $v_{0}=v_{5}$ ). Then $x_{i} \notin\left\{v_{1}, \ldots, v_{5}\right\}$, because $G$ is triangle-free. Assume that

- the vertices $x_{1}, x_{2}, x_{3}, x_{4}$ are pairwise distinct and pairwise non-adjacent, and

- there is no path in $G \backslash\left\{v_{1}, v_{2}, v_{3}, v_{4}\right\}$ of length at most three from $x_{2}$ to $v_{5}$, and

- every path in $G \backslash\left\{v_{1}, v_{2}, v_{3}, v_{4}\right\}$ of length at most three from $x_{3}$ to $x_{4}$ has length exactly two, and its completion via the path $x_{3} v_{3} v_{4} x_{4}$ results in a facial cycle of length five in $G$ (in particular, there is at most one such path).

In those circumstances we say that the pentagram $\left(v_{1}, v_{2}, \ldots, v_{5}\right)$ is safe.

Lemma 2.2. Every triangle-free plane graph $G$ of minimum degree at least three has a safe tetragram, a safe pentagram, or a safe hexagram.

Proof. Let $G$ be as stated. If $\left(v_{1}, v_{2}, v_{3}, v_{4}\right)$ is a tetragram in $G$, then one of the tetragrams $\left(v_{1}, v_{2}, v_{3}, v_{4}\right),\left(v_{2}, v_{3}, v_{4}, v_{1}\right)$ is safe, as $G$ is planar and triangle-free. Thus we may assume that $G$ has no 4 -faces, and hence every 4-cycle in $G$ is separating.

Let us define an induced subgraph $G_{1}$ of $G$ and a facial cycle $C_{1}$ of $G_{1}$ in the following way: If $G$ has a separating cycle of length at most five, then let 
us select such a cycle $C_{1}$ so that the disk it bounds is as small as possible, and let $G_{1}$ be the subgraph of $G$ consisting of all vertices and edges drawn in the closed disk bounded by $C_{1}$. If $G$ has no separating cycle of length at most five, then let $G_{1}:=G$ and let $C_{1}$ be a facial cycle of $G$ of length at most five. Such a facial cycle exists, because the minimum degree of $G$ is at least three. In the latter case, we also redraw $G$ so that $C_{1}$ becomes the outer face; thus $G_{1}$ is always drawn in the closed disk bounded by $C_{1}$. Note that $G_{1}$ does not contain any separating cycle of length at most five, and thus $G_{1}$ does not contain any 4-cycle except possibly $C_{1}$.

Next, we define a subgraph $G_{2}$ of $G_{1}$ and its facial cycle $C_{2}$ as follows. If $G_{1}$ contains a separating cycle of length six, then choose such a cycle $C_{2}$ so that the disk it bounds contains as few vertices as possible, and let $G_{2}$ be the subgraph of $G_{1}$ consisting of all vertices and edges drawn in the closed disk bounded by $C_{2}$. Otherwise, let $G_{2}:=G_{1}$ and $C_{2}:=C_{1}$. Note that $G_{2}$ does not contain any separating cycle of length at most six. As $G$ has no 4 -faces, it follows that any cycle of length at most six in $G_{2}$ bounds a face.

The cycle $C_{2}$ is induced in $G$, for if it had a chord, then the chord would belong to $G_{1}$ (because $G_{1}$ is an induced subgraph of $G$ ), and hence $V\left(C_{2}\right)$ would include the vertex-sets of two distinct cycles of length at most (and hence exactly) four in $G_{1}$, a contradiction.

From Lemma 2.1 applied to the graph $G_{2}$ and facial cycle $C_{2}$ we deduce that $G_{2}$ has a pentagram $\left(v_{1}, v_{2}, v_{3}, v_{4}, v_{5}\right)$ such that $v_{1}, v_{2}, v_{3}, v_{4} \notin V\left(C_{2}\right)$. We may assume that neither this pentagram nor the pentagram $\left(v_{4}, v_{3}, v_{2}, v_{1}, v_{5}\right)$ is safe in $G$, for otherwise the lemma holds. Let $x_{i}$ be the neighbor of $v_{i}$ outside of the pentagram, for $1 \leq i \leq 4$. Note that all of these neighbors belong to $G_{2}$, and as $G_{2}$ is triangle-free and contains no 4-cycles other than $C_{2}$ and no separating cycles of length at most 5, they are distinct and mutually non-adjacent. It follows that $\left|\left\{x_{1}, x_{2}, x_{3}, x_{4}\right\} \cap V\left(C_{2}\right)\right| \leq 3$, and by symmetry we may assume that at least one of $x_{3}$ and $x_{4}$ does not lie on $C_{2}$. Furthermore, as each cycle of length at most six in $G_{2}$ is facial, if $v_{5} \in V\left(C_{2}\right)$, then $\left\{x_{1}, x_{2}, x_{3}, x_{4}\right\} \cap V\left(C_{2}\right)=\emptyset$.

Since the pentagram $\left(v_{1}, v_{2}, v_{3}, v_{4}, v_{5}\right)$ is not safe in $G$, there exists a pair of vertices $x, y$ such that either $\{x, y\}=\left\{x_{2}, v_{5}\right\}$ or $\{x, y\}=\left\{x_{3}, x_{4}\right\}$, and there exists a path $P$ in $G \backslash\left\{v_{1}, v_{2}, v_{3}, v_{4}\right\}$ with ends $x$ and $y$ such that $P$ has length at most three, and if $\{x, y\}=\left\{x_{3}, x_{4}\right\}$, then either $P$ has length exactly three, or its completion via the path $x_{3} v_{3} v_{4} x_{4}$ does not result in a facial cycle in $G$. If $\{x, y\}=\left\{x_{2}, v_{5}\right\}$ then let $Q$ denote the path $x_{2} v_{2} v_{1} v_{5}$; otherwise let $Q$ denote the path $x_{3} v_{3} v_{4} x_{4}$. Suppose first that $P \cup Q$ bounds 
a face in $G$. Then it follows that $\{x, y\}=\left\{x_{3}, x_{4}\right\}$, and hence $P$ has length exactly three. Let the vertices of $P \cup Q$ be $x_{3} v_{3} v_{4} x_{4} a b$ in order. Let us argue that $\left(x_{4}, v_{4}, v_{3}, x_{3}, a, b\right)$ is a safe hexagram. If that were not the case, then there would exist a path $x_{4} u_{1} v_{3}$ or $x_{4} u_{1} u_{2} v_{3}$ for some $u_{1}, u_{2} \neq v_{4}$. Since $v_{2}$ and $v_{3}$ have degree three and the vertices $x_{1}, x_{2}, x_{3}$ and $x_{4}$ are distinct and mutually non-adjacent, the former case is not possible, and in the latter case $u_{2}=x_{3}$. However, since at most one of $x_{3}$ and $x_{4}$ lies on $C_{2}, x_{4} u_{1} x_{3} v_{3} v_{4}$ would be a separating 5 -cycle in $G_{2}$, and hence in $G_{1}$, a contradiction.

Thus we may assume that $P \cup Q$ does not bound a face in $G$, and so $P \cup Q$ is a separating cycle in $G$. It follows from the choice of $C_{2}$ that $P \cup Q$ is not a subgraph of $G_{2}$. But not both $x, y$ belong to $C_{2}$ and $C_{2}$ is induced; thus a subpath $R$ of $P \cup Q$ of length four joins two vertices $w_{1}, w_{4}$ of $C_{2}$, and a vertex $w$ of $(P \cup Q) \backslash V\left(G_{2}\right)$ is adjacent to both $w_{1}$ and $w_{4}$. If $w \notin V\left(G_{1}\right)$, then $w_{1}, w_{4} \in V\left(C_{1}\right)$, because they belong to $C_{2}$. But $C_{1}$ has length at most five, and $w_{1}, w_{4}$ are not adjacent, because $G$ is triangle-free. Thus $w_{1}, w_{4}$ have a common neighbor in $C_{1}$, and this neighbor can replace $w$. Thus we may assume that $w \in V\left(G_{1}\right)$.

If $w_{1}$ and $v_{4}$ have a common neighbor in $C_{2}$, then $R$ can be completed using this neighbor to a cycle that contradicts the choice of $C_{2}$. It follows that $w_{1}, w_{4}$ are at distance three on $C_{2}$, and so we may assume that the vertices of $C_{2}$ are $w_{1}, w_{2}, \ldots, w_{6}$, in order. From the symmetry we may assume that $w_{1} w_{2} w_{3} w_{4} w$ bounds a face, by the minimality of $C_{1}$. Thus the closed disk bounded by $P \cup Q$ does not include $w_{5}, w_{6}$, and it includes no vertex of $V(G)-V\left(G_{2}\right)$, except $w$. Thus $P \cup Q$ contradicts the choice of $C_{2}$.

Proof of Theorem 1.1. Let $G$ be a triangle-free plane graph. We proceed by induction on $|V(G)|$. We may assume that every vertex $v$ of $G$ has degree at least three, for otherwise the theorem follows by induction applied to $G \backslash v$. By Lemma 2.2 there is a safe tetra-, penta-, or hexagram $\left(v_{1}, v_{2}, \ldots, v_{k}\right)$. If $k=4$ or $k=6$, then we apply induction to the graph obtained from $G$ by identifying $v_{1}$ and $v_{3}$. It follows from the definition of safety that the new graph has no triangles, and clearly every 3 -coloring of the new graph extends to a 3-coloring of $G$.

Thus we may assume that $\left(v_{1}, v_{2}, \ldots, v_{5}\right)$ is a safe pentagram in $G$. Let $G^{\prime}$ be obtained from $G \backslash\left\{v_{1}, v_{2}, v_{3}, v_{4}\right\}$ by identifying $v_{5}$ with $x_{2}$, and $x_{3}$ with $x_{4}$. It follows from the definition of safety that $G^{\prime}$ is triangle-free, and hence it is 3-colorable by the induction hypothesis. Any 3-coloring of $G^{\prime}$ can be extended to a 3-coloring of $G$ : let $c_{1}$ be the color of $x_{1}, c_{2}$ the color of $x_{2}$ 
and $v_{5}$, and $c_{3}$ the color of $x_{3}$ and $x_{4}$. If $c_{1}=c_{2}$, then we color the vertices $v_{4}, v_{3}, v_{2}$ and $v_{1}$ in this order. Note that when $v_{i}(i=1,2,3,4)$ is colored, it is adjacent to vertices of at most two different colors, and hence we can choose the third color for it. Similarly, if $c_{2}=c_{3}$, then we color the vertices in the following order: $v_{1}, v_{2}, v_{3}$ and $v_{4}$. Let us now consider the case that $c_{1} \neq c_{2} \neq c_{3}$. We color $v_{2}$ with $c_{1}, v_{3}$ with $c_{2}$, and choose a color different from $c_{1}$ and $c_{2}$ for $v_{1}$ and a color different from $c_{2}$ and $c_{3}$ for $v_{4}$. Thus $G$ is 3-colorable, as desired.

Let us note that the essential ideas of the proof came from Thomassen's work [15]. For graphs of girth at least five Thomassen actually proves a stronger statement, namely that every 3-coloring of an induced facial cycle of length at most nine extends to a 3-coloring of the entire triangle-free plane graph, unless some vertex of $G$ has three distinct neighbors on $C$ (and those neighbors received three different colors). By restricting ourselves to Theorem 1.1 we were able to somewhat streamline the argument. Another variation of the same technique is presented in [7].

\section{Graph representation}

For the purpose of our algorithm, graphs will be represented by means of doubly linked adjacency lists. More precisely, the neighbors of each vertex $v$ will be listed in the clockwise cyclic order in which they appear around $v$, and the two occurrences of the same edge will be linked to each other. The facial walks of the graph can be read off from this representation using the standard face tracing algorithm (Mohar and Thomassen [9], page 93). Thus all vertices and edges incident with a facial cycle of length $k$ can be listed in time $O(k)$. Here we make use of our non-standard definition of facial cycle.

Suppose that $D$ is a fixed constant (in our algorithm, $D=59$ ). We can perform the following operations with graphs represented in the described way in constant time:

- remove an edge when a corresponding entry of the adjacency list is given

- add an edge with ends $u, v$ into a face $f$, assuming that the edges preceding and following $u, v$ in the facial boundary of $f$ are specified

- remove an isolated vertex 
- determine the degree of a vertex $v$ if $\operatorname{deg}(v) \leq D$, or prove that $\operatorname{deg}(v)>$ $D$

- check whether two vertices $u$ and $v$ such that $\min (\operatorname{deg}(u), \operatorname{deg}(v)) \leq D$ are adjacent

- check whether the distance between two vertices $u$ and $v$ such that $\max (\operatorname{deg}(u), \operatorname{deg}(v)) \leq D$ is at most two

- given an edge $e$ incident with a face $f$, output all vertices whose distance from $e$ in the facial walk of $f$ is at most two, and determine whether the length of the component of the boundary of $f$ that contains $e$ has length at most 6

- output the subgraph consisting of vertices reachable from a vertex $v_{0}$ through a path $v_{0}, v_{1}, \ldots, v_{t}$ of length $t \leq D$, such that $\operatorname{deg}\left(v_{i}\right) \leq D$ for $0 \leq i<t$ (but the degree of $v_{t}$ may be arbitrary).

All the transformations and queries executed in the algorithm can be expressed in terms of these simple operations.

\section{The algorithm}

The idea of our algorithm is to find a safe tetragram, pentagram or hexagram $\gamma$ in $G$ and use it to reduce the size of the graph as in the proof of Theorem 1.1 above. Finding $\gamma$ is easy, but the difficulty lies in testing safety. To resolve this problem we prove a variant of Lemma 2.2 that will guarantee the existence of such $\gamma$ with an additional property that will allow testing safety in constant time. The additional property, called security, is merely that enough vertices in and around $\gamma$ have bounded degree. Unfortunately, the additional property we require necessitates the introduction of two more configurations, a variation of tetragram called "octagram" and a variation of pentagram called "decagram". For the sake of consistency, we say that a monogram in a graph $G$ is the one-vertex sequence $(v)$ comprised of a vertex $v \in V(G)$ of degree at most two.

Now let $G$ be a plane graph, let $k \in\{1,4,5,6\}$ and let $\gamma=\left(v_{1}, v_{2}, \ldots, v_{k}\right)$ be a mono-, tetra-, penta-, or hexagram in $G$. Let $C$ be a subgraph of $G$. (For the purpose of this section the reader may assume that $C$ is the null graph, but in the next section we will need $C$ to be a facial cycle of $G$.) A 
vertex of $G$ is big if it has degree at least 60 , and small otherwise. A vertex $v \in V(G)$ is $C$-admissible if it is small and does not belong to $C$; otherwise it is $C$-forbidden. A pentagram $\left(v_{1}, v_{2}, \ldots, v_{5}\right)$ is called a decagram if $v_{5}$ has degree exactly three (and hence $v_{1}, \ldots, v_{5}$ all have degree three). A tetragram is called an octagram if all its vertices have degree exactly three. A multigram is a monogram, tetragram, pentagram, hexagram, octagram or a decagram. The vertex $v_{1}$ will be called the pivot of the multigram $\left(v_{1}, v_{2}, \ldots, v_{k}\right)$. In the following $\gamma$ will be a multigram, and we will define (or recall) what it means for $\gamma$ to be safe and $C$-secure. We will also define a smaller graph $G^{\prime}$, which will be called the $\gamma$-reduction of $G$.

If $\gamma$ is a monogram, then we define it to be always safe, and we say that it is $C$-secure if $v_{1} \notin V(C)$. We define $G^{\prime}:=G \backslash v_{1}$.

Now let $\gamma$ be a tetragram. Let us recall that $\gamma$ is safe if the only paths in $G$ of length at most three with ends $v_{1}$ and $v_{3}$ are subgraphs of the facial cycle $v_{1} v_{2} v_{3} v_{4}$. We say that $\gamma$ is $C$-secure if

- it is safe, and

- $v_{1}$ is $C$-admissible and has degree exactly three, and

- letting $x$ denote the neighbor of $v_{1}$ other than $v_{2}$ and $v_{4}$, the vertex $x$ is $C$-admissible, and

- either

- $v_{3}$ is $C$-admissible, or

- every neighbor $w$ of $x$ is $C$-admissible or belongs to a 4-face incident with the edge $v_{1} x$ (either $v_{1} v_{2} w x$ or $v_{1} v_{4} w x$ ).

We define $G^{\prime}$ to be the graph obtained from $G$ by identifying the vertices $v_{1}$ and $v_{3}$ and deleting one edge from each of the two pairs of parallel edges that result.

If $\gamma$ is an octagram, then it is always safe, and it is $C$-secure if $v_{1}, v_{2}, v_{3}, v_{4}$ are all are $C$-admissible. We define $G^{\prime}:=G \backslash\left\{v_{1}, v_{2}, v_{3}, v_{4}\right\}$.

Now let $\gamma$ be a decagram, and for $i=1,2,3,4$ let $x_{i}$ be the neighbor of $v_{i}$ other than $v_{i-1}$ or $v_{i+1}$, where $v_{0}$ means $v_{5}$. We say that the decagram $\gamma$ is safe if $x_{1}, x_{3}$ are distinct, non-adjacent and there is no path of length two between them. We say that $\gamma$ is $C$-secure if it is safe and the vertices $v_{1}, v_{2}, \ldots, v_{5}, x_{1}, x_{3}$ are all $C$-admissible. We define $G^{\prime}$ to be the graph obtained from $G \backslash\left\{v_{1}, v_{2}, \ldots, v_{5}\right\}$ by adding the edge $x_{1} x_{3}$. 
Now let $\gamma$ be a pentagram, and for $i=1,2,3,4$ let $x_{i}$ be as in the previous paragraph. Let us recall that the safety of $\gamma$ was defined prior to Lemma 2.2. We say that $\gamma$ is $C$-secure if it is safe, the vertices $v_{1}, v_{2}, \ldots, v_{5}, x_{1}, x_{2}, x_{3}, x_{4}$ are all $C$-admissible, either $v_{5}$ or $x_{2}$ has no $C$-forbidden neighbor, and either $x_{3}$ or $x_{4}$ has no $C$-forbidden neighbor. We define $G^{\prime}$ as in the proof of Theorem 1.1: $G^{\prime}$ is obtained from $G \backslash\left\{v_{1}, v_{2}, v_{3}, v_{4}\right\}$ by identifying $x_{2}$ and $v_{5}$; identifying $x_{3}$ and $x_{4}$; and deleting one of the parallel edges should $x_{3}$ and $x_{4}$ have a common neighbor.

Finally, let $\gamma$ be a hexagram. Let us recall that $\gamma$ is safe if every path of length at most three in $G$ between $v_{1}$ and $v_{3}$ is the path $v_{1} v_{2} v_{3}$. We say that $\gamma$ is $C$-secure if $v_{1}, v_{3}, v_{6}$ are $C$-admissible, $v_{1}$ has degree exactly three, and the neighbor of $v_{1}$ other than $v_{2}$ or $v_{6}$ is $C$-admissible. We define $G^{\prime}$ to be the graph obtained from $G$ by identifying the vertices $v_{1}$ and $v_{3}$ and deleting one of the parallel edges that result.

We say that a multigram $\gamma$ is secure if it is $K_{0}$-secure, where $K_{0}$ denotes the null graph. This completes the definition of safe and secure multigrams.

Lemma 4.1. Let $G$ be a triangle-free plane graph, let $\gamma$ be a safe multigram in $G$, and let $G^{\prime}$ be the $\gamma$-reduction of $G$. Then $G^{\prime}$ is triangle-free, and every 3-coloring of $G^{\prime}$ can be converted to a 3-coloring of $G$ in constant time. Moreover, if $\gamma$ is secure, then $G^{\prime}$ can be regarded as having been obtained from $G$ by deleting at most 126 edges, adding at most 116 edges, and deleting at least one isolated vertex.

Proof. The graph $G^{\prime}$ is triangle-free, because $\gamma$ is safe. As in the proof of Theorem 1.1, we argue that every 3-coloring of $G^{\prime}$ can be extended to a 3coloring of $G$. If $\gamma$ is secure, then every time vertices $u$ and $v$ are identified in the construction of $G^{\prime}$, one of $u, v$ is small. Thus the identification of $u$ and $v$ can be seen as a deletion of at most 59 edges and addition of at most 59 edges. The lemma follows by a more careful examination of the construction of $G^{\prime}$.

Let $G$ and $C$ be as above. We say that two small vertices $u, v \in V(G)$ are close if either there is a path of length at most four between $u$ and $v$ consisting of small vertices, or a facial cycle of length at most six contains both $u$ and $v$. A vertex $u$ is close to an edge $e$ if both $u$ and $e$ belong to the facial walk of the same face and the distance between $u$ and and one end of $e$ in this facial walk is at most two. Thus for every vertex $v$ there are at most 
$1+4 \cdot 59+59^{2}+59^{3}+59^{4}$ vertices that are close to $v$, and for every edge $e$, there are at most 10 vertices that are close to $e$.

Lemma 4.2. Given a triangle-free plane graph $G$ and a vertex $v \in V(G)$, it can be decided in constant time whether $G$ has a secure multigram with pivot $v$.

Proof. This follows by inspecting the subgraph of $G$ induced by vertices and edges that are close to $v$ and testing the security of all multigrams with pivot $v$ that lie in this subgraph. Given such multigram, the only non-trivial part of testing security is testing safety. Thus we may assume that the multigram satisfies all conditions in the definition of security, except safety. To test safety we need to check the existence of certain paths $P$ of bounded length with prescribed ends. We claim that whenever such a test is needed every vertex of $P$, except possibly one, is small. The claim follows easily, except in the case of a tetragram $v v_{2} v_{3} v_{4}$, where $v$ has degree three, the vertex $v_{3}$ is big, and letting $x$ denote the neighbor of $v_{1}$ other than $v_{2}$ and $v_{4}, x$ is small, but has a big neighbor $w$. In this case the straightforward check whether $w$ and $x_{3}$ are adjacent would take more than constant time, but it actually follows that $w$ and $x_{3}$ are not adjacent: the vertex $w$ belongs to a 4 -face incident with the edge $v x$, for otherwise the tetragram is not secure; but then it follows that $w$ and $x_{3}$ are not adjacent, for otherwise $w v_{3} v_{2}$ would be a triangle. This proves our claim that in the course of testing safety it suffices to examine paths with all but one vertex small.

It follows from the claim that security can be tested in constant time, as desired.

Lemma 4.3. Let $G$ and $G^{\prime}$ be triangle-free plane graphs, such that for some pair of non-adjacent vertices $u, v \in V(G)$ the graph $G^{\prime}$ is obtained from $G$ by adding the edge uv. Let $\gamma$ be a secure multigram in exactly one of the graphs $G, G^{\prime}$. Then the pivot of $\gamma$ is close to u or $v$ in $G$, or to the edge uv in $G^{\prime}$.

Proof. Let $v_{1}$ be the pivot of $\gamma$. The claim is obvious if $v_{1} \in\{u, v\}$, and thus assume this is not the case. In particular, $\gamma$ is not a monogram or an octagram, and $\gamma$ corresponds to a facial cycle $F$ in $G$ or $G^{\prime}$. If $F$ does not exist in $G$ or $F$ is not facial in $G$ or $G^{\prime}$, then $v_{1}$ is close to the edge $u v$ in $G^{\prime}$. Let us now consider the case that $F$ is a facial cycle both in $G$ and $G^{\prime}$. As $v_{1} \notin\{u, v\}$, the degree of $v_{1}$ is three both in $G$ and $G^{\prime}$. Let $x_{1}$ be the neighbor of $v_{1}$ distinct from its neighbors on $F$. Note that $x_{1}$ is small in $G$. 
Suppose first that $\gamma$ is a tetragram or a hexagram. Observe that the removal of the edge $u v$ from $G^{\prime}$ must decrease the degree of some of the vertices affecting the security of $\gamma$, change the length of one of the faces incident with the edge $v_{1} x_{1}$ affecting the security of $\gamma$, or destroy a path affecting its safety. Therefore, if $\{u, v\} \cap\left(V(F) \cup\left\{x_{1}\right\}\right)=\emptyset$ and $v_{1}$ is not close to the edge $u v$ in $G^{\prime}$, then $u$ or $v$ is a small neighbor of $x_{1}$ in $G$ that is big in $G^{\prime}$. We conclude that $v_{1}$ is close to $u$ or $v$ in $G$.

Let us now consider the case that $\gamma=\left(v_{1}, v_{2}, \ldots, v_{5}\right)$ is a decagram or a pentagram. As $\gamma$ is secure in $G$ or $G^{\prime}$, all the vertices of $\gamma$ are small in $G$. If $\{u, v\} \cap V(F) \neq \emptyset$, then $v_{1}$ is close to $u$ or $v$ in $G$, and thus assume that this is not the case. It follows that the degree of $v_{i}$ is the same in $G$ and $G^{\prime}$, for $1 \leq i \leq 5$; in particular, $\operatorname{deg}\left(v_{i}\right)=3$ for $1 \leq i \leq 4$. Let $x_{i}$ be the neigbor of $v_{i}$ not incident with $F$, for $1 \leq i \leq 4$. Similarly, we conclude that $x_{1}$ and $x_{3}$ are small in $G$, and if $\gamma$ is a pentagram, then $x_{2}$ and $x_{4}$ are small in $G$. If $\{u, v\} \cap\left\{x_{1}, x_{3}\right\} \neq \emptyset$, or $\gamma$ is a pentagram and $\{u, v\} \cap\left\{x_{2}, x_{4}\right\} \neq \emptyset$, then $u$ or $v$ is close to $v_{1}$ in $G$. If this is not the case, then the removal or addition of $u v$ cannot affect the security of $\gamma$ if $\gamma$ is a decagram.

We are left with the case when $\gamma$ is a pentagram, and $\{u, v\} \cap\left\{x_{1}, x_{2}, x_{3}, x_{4}\right\}=$ $\emptyset$. It follows that the neighborhoods of $x_{2}, x_{3}, x_{4}$ and $v_{5}$ are the same in $G$ and in $G^{\prime}$. As $\gamma$ is secure in $G$ or $G^{\prime}$, all neighbors of $v_{5}$ or $x_{2}$, and all neighbors of $x_{3}$ or $x_{4}$ are small in $G$. As $\gamma$ is not secure both in $G$ and $G^{\prime}$, the removal of $u v$

- destroys a path of length at most three between $x_{2}$ and $v_{5}$ or between $x_{3}$ and $x_{4}$, or

- removes an edge incident with the common neighbor $y$ of $x_{3}$ and $x_{4}$, thus making the 5 -cycle $x_{3} v_{3} v_{4} x_{4} y$ facial, or

- decreases the degree of a neighbor of $x_{2}, x_{3}, x_{4}$ or $v_{5}$, making it small in $G$.

In all the cases, $u$ or $v$ is a small neighbor of $x_{2}, x_{3}, x_{4}$ or $v_{5}$, and hence it is close to $v_{1}$ in $G$.

The next theorem will serve as the basis for the proof of correctness of our algorithm. We defer its proof until the next section.

Theorem 4.4. Every non-null triangle-free planar graph has a secure multigram. 
We are now ready to prove Theorem 1.2 , assuming Theorem 4.4 .

Algorithm 4.5. There is an algorithm with the following specifications: Input: A triangle-free planar graph.

Output: A proper 3-coloring of $G$.

Running time: $O(|V(G)|)$.

Description. Using a linear-time planarity algorithm that actually outputs an embedding, such as [13] or [18], we can assume that $G$ is a plane graph. The algorithm is recursive. Throughout the execution of the algorithm we will maintain a list $L$ that will include the pivots of all secure multigrams in $G$, and possibly other vertices as well. We initialize the list $L$ to consist of all vertices of $G$ of degree at most three.

At a general step of the algorithm we remove a vertex $v$ from $L$. There is such a vertex by Theorem 4.4 and the requirement that $L$ include the pivots of all secure multigrams. We check if $G$ has a secure multigram with pivot $v$. This can be performed in constant time by Lemma 4.2, If no such multigram exists, then we go to the next iteration. Otherwise, we let $\gamma$ be one such multigram, and let $G^{\prime}$ be the $\gamma$-reduction of $G$. By Lemma 4.1 the graph $G^{\prime}$ is triangle-free and can be constructed in constant time by adding and deleting bounded number of edges, and removing a bounded number of isolated vertices. For every edge $u v$ that was deleted or added during the construction of $G^{\prime}$ we add to $L$ all vertices that are close to $u$ or $v$, or to the edge $u v$ in $G$ or $G^{\prime}$. By Lemma 4.3 this will guarantee that $L$ will include the pivots of all secure multigrams in $G^{\prime}$. We apply the algorithm recursively to $G^{\prime}$, and convert the resulting 3-coloring of $G^{\prime}$ to one of $G$ using Lemma 4.1. Since the number of vertices added to $L$ is proportional to the number of vertices removed from $G$ we deduce that the number of vertices added to $L$ (counting multiplicity) is at most linear in the number of vertices of $G$. Thus the running time is $O(|V(G)|)$, as claimed.

Algorithm 4.5 has the following extension.

Algorithm 4.6. There is an algorithm with the following specifications: Input: A triangle-free plane graph $G$, a facial cycle $C$ in $G$ of length at most five, and a proper 3 -coloring $\phi$ of $C$.

Output: A proper 3-coloring of $G$ whose restriction to $V(C)$ is equal to $\phi$. Running time: $O(|V(G)|)$. 
Description. The description is exactly the same, except that we replace "secure" by "C-secure" and appeal to Lemma 5.1 rather than Theorem 4.4.

\section{Proof of correctness}

In this section we prove Theorem 4.4, thereby completing the proof of correctness of the algorithm from the previous section. The theorem will follow from the next lemma. If $x y$ is an edge in a plane graph, and $f$ is a face of $G$ incident with $y$ but not with the edge $x y$, then we say that $f$ is opposite to $x y$. Let us emphasize that this notion is not symmetric in $x, y$.

Lemma 5.1. Let $G$ be a connected triangle-free plane graph and let $f_{0}$ be its outer face. Assume that $f_{0}$ is bounded by a cycle $C$ of length at most six, $V(G) \neq V(C)$, and if $C$ has length six, then $|V(G)-V(C)| \geq 2$. Then $G$ contains a $C$-secure multigram.

Proof. Suppose for a contradiction that the lemma is false, and let $G$ be a counterexample with $|E(G)|$ minimum. We first establish the following claim.

(1) If $K \neq C$ is a cycle in $G$ of length at most six, then $K$ bounds a face, or $K$ has length six and the open disk bounded by $K$ contains at most one vertex.

To prove (1) let $K$ be as stated, and let $G^{\prime}$ be the subgraph of $G$ consisting of all vertices and edges that belong to the closed disk bounded by $K$. If $K$ does not satisfy the conclusion of (1), then $G^{\prime}$ and $K$ satisfy assumptions of Lemma 5.1. From the induction hypothesis applied to $G^{\prime}$ and $K$ we deduce that $G^{\prime}$ has a $K$-secure multigram. However, every $K$-secure multigram in $G^{\prime}$ is a $C$-secure multigram in $G$.

It follows from (1) that $C$ is an induced cycle and that every tetragram in $G$ is safe.

We assign charges to vertices and faces of $G$ as follows. Initially, a vertex $v$ will receive a charge of $9 \operatorname{deg}(v)-36$ if $v \notin V(C)$, and $8 \operatorname{deg}(v)-19$ otherwise. The outer face $f_{0}$ will receive a charge of zero, and every other face $f$ of length 
$\ell$ will receive a charge of $9 \ell-36$. By Euler's formula the sum of the charges is equal to

$$
\begin{aligned}
& \sum_{v \notin V(C)} 9(\operatorname{deg}(v)-4)+\sum_{v \in V(C)}(8 \operatorname{deg}(v)-19)+\sum_{f \neq f_{0}} 9(\operatorname{size}(f)-4) \\
= & \sum_{v \in V(G)} 9(\operatorname{deg}(v)-4)+\sum_{f} 9(\operatorname{size}(f)-4)-\sum_{v \in V(C)} \operatorname{deg}(v)+8|V(C)|+36 \\
= & 8|V(C)|-\sum_{v \in V(C)} \operatorname{deg}(v)-36 \leq-1,
\end{aligned}
$$

because all vertices of $C$ have degree at least two, and at least one has degree at least three by hypothesis. Furthermore,

(2) if at least $k$ vertices of $C$ have degree at least three, then the sum of the charges is at most $-k$.

We now redistribute the charges according to the following rules. The new charge thus obtained will be referred to as the final charge. We need a definition first. Let $f \neq f_{0}$ be a face of $G$ incident with a vertex $v \in V(C)$. If there exist two consecutive edges in the boundary of $f$ such that both are incident with $v$ and neither belongs to $C$, then we say that $f$ is a $v$-interior face. The rules are:

(A) every face other than $f_{0}$ sends three units of charge to every incident vertex $v$ such that either $v \in V(C)$ and $v$ has degree two in $G$, or $v \notin V(C)$ and $v$ has degree exactly three,

(B) every big vertex not on $C$ sends three units to each incident face, and four units to each 4-face that shares an edge with $C$,

(C) every vertex $v \in V(C)$ sends three units to every $v$-interior face,

(D) if $x \in V(G)$ is $C$-forbidden, and $y$ is a $C$-admissible neighbor of $x$ of degree three, then $x$ sends three units to the unique face opposite to $x y$, and one unit to the face opposite to $y z$ for every $C$-admissible neighbor $z$ of $y$ of degree three,

(E) every $C$-forbidden vertex sends five units to every $C$-admissible neighbor of degree at least four, 
(F) for every $C$-admissible vertex $y$ of degree at least four that has a $C$ forbidden neighbor we select a $C$-forbidden neighbor $x$ of $y$ and let $y$ send one unit to each face opposite to $x y$, and one unit to the face opposite to $y z$ for every $C$-admissible neighbor $z$ of $y$ of degree three.

Since $G$ does not satisfy the conclusion of the theorem, it follows that every vertex of $G$ has degree at least two, and every vertex of degree exactly two belongs to $C$. With these facts in mind we now show that every vertex has non-negative charge. To that end let $v \in V(G)$ have degree $d$, and assume first that $v$ is $C$-admissible. If $d=3$, then it starts out with a charge of -9 and receives three from each incident face by rule (A) for a final total of zero. If $d \geq 4$, then $v$ starts out with a charge of $9 d-36 \geq 0$. If $v$ has no $C$ forbidden neighbor, then it sends no charge and the claim holds. Thus we may assume that $v$ has a $C$-forbidden neighbor, and let $x$ be such neighbor selected by rule $(\mathrm{F})$. Then $v$ receives at least five units by rule $(\mathrm{E})$, and sends at most $2 d-3$ by rule $(\mathrm{F})$ for a total of at least $9 d-36+5-(2 d-3)=7 d-28 \geq 0$. Thus every $C$-admissible vertex has non-negative final charge. If $v$ is big, but does not belong to $C$, then it sends only by rules (B), (D) or (E). It sends at most $3 d$ using the first clause of rule (B), at most 24 using the second clause of rule (B) and at most $5 d$ using rules (D) or (E) for a total final charge of at least $9 d-36-3 d-24-5 d \geq 0$, because $d \geq 60$. Thus we may assume that $v \in V(C)$. Then $v$ starts out with a charge of $8 d-19$ and sends a net total of $3(d-3)$ using rules (A) or (C) (if $d=2$, then $v$ receives 3 by rule (A); and otherwise it sends $3(d-3)$ by rule $(\mathrm{C}))$ and it sends $5(d-2)$ using rule (D) or (E) for a total of $8 d-19-3(d-3)-5(d-2)=0$. This proves our claim that the final charge of every vertex is non-negative.

It also follows that every face of length $\ell \geq 6$ has non-negative final charge, for every face sends at most three units to each incident vertex and only to those vertices by rule (A); thus the final charge is at most $9 \ell-36-3 \ell \geq 0$.

We have thus shown that $G$ has a face $f$ of length at most five with strictly negative final charge. Clearly $f$ is not the outer face.

(3) No vertex incident with $f$ has degree two.

To prove (3) suppose for a contradiction that a vertex $v$ of degree two is incident with $f$. Thus $v$ and the two edges incident with $v$ and $f$ belong to $C$. Since $G \neq C$ and $f$ has length at most five we deduce that at least two vertices incident with $f$ are incident with $C$ and have degree at least three. 
Those two vertices do not receive any charge from $f$, and hence $f$ has length four, because it has negative charge.

We deduce that $f$ is bounded by a cycle $u_{1} u_{2} u_{3} u_{4}$, where $u_{1}, u_{2}, u_{3}$ are consecutive vertices of $C$, and $u_{2}$ has degree two. It follows that $u_{4} \notin V(C)$, because $C$ is induced. Since $f$ has negative charge it does not receive charge by rule (B), and hence $u_{4}$ is small and $C$-admissible. Let $C^{\prime}$ be the cycle obtained from $C$ by replacing the vertex $u_{2}$ by $u_{4}$; note that $\left|V\left(C^{\prime}\right)\right|=$ $|V(C)| \leq 6$. As $u_{4}$ has degree greater than two, $C^{\prime}$ does not bound a face, hence it follows from (1) that $\left|V\left(C^{\prime}\right)\right|=6$ and the open disk bounded by $C^{\prime}$ contains at most one vertex. Therefore, it contains exactly one, because $|V(G)|-V(C) \mid \geq 2$. Let that vertex be $v_{4}$; then the remaining vertices of $C$ can be numbered $v_{1}, v_{2}, v_{3}$ so that the cycle $C$ is $u_{1} u_{2} u_{3} v_{1} v_{2} v_{3}$ and $v_{4}$ is adjacent to $v_{1}, v_{3}$ and $u_{4}$. Then $\left(u_{4}, u_{1}, u_{2}, u_{3}\right)$ is a $C$-secure tetragram, contrary to the assumption that $G$ is a counterexample to the theorem. This proves (3).

Let $u v$ be an edge of $G$ such that $f$ is opposite to $u v$. Let us say that $v$ is a sink if $v$ has degree three and both $u$ and $v$ are $C$-admissible. Let us say that $v$ is a source if either $v \notin V(C)$ and $v$ is big, or $v \in V(C)$ and $f$ is $v$-interior. Since $v$ does not have degree two by (3) we deduce that $v$ is a sink if and only if it has degree three and receives three units of charge from $f$ by rule (A) and $f$ does not receive three units by rule (D) from $u$. Likewise, the vertex $v$ is a source if and only if it sends three units to $f$ by the first clause of rule $(\mathrm{B})$ or by rule $(\mathrm{C})$. Let $s$ be the number of sources, and $t$ the number of sinks. Thus the charge of $f$ is at least $9+3 s-3 t$ if $f$ has length five and at least $3 s-3 t$ if $f$ has length four.

Let us assume now that $f$ has length five, and let $v_{1}, v_{2}, \ldots, v_{5}$ be the incident vertices, listed in order. Since $f$ has negative charge, at least four of the five incident vertices are sinks, and so we may assume that $v_{1}, v_{2}, v_{3}, v_{4}$ are sinks. Thus $\gamma=\left(v_{1}, v_{2}, \ldots, v_{5}\right)$ is a pentagram. For $i=1,2,3,4$ let $x_{i}$ be the neighbor of $v_{i}$ distinct from $v_{i-1}$ and $v_{i+1}$ (where $v_{0}=v_{5}$ ). From (1) and the fact that $G$ has no $C$-secure tetragram we deduce that the vertices $x_{1}, x_{2}, x_{3}, x_{4}$ are distinct and pairwise non-adjacent. If $v_{5}$ is a $C$-admissible vertex of degree three, then it follows from (1) that $\gamma$ is $C$-secure decagramotherwise, if there is a path of length two between $x_{1}$ and $x_{3}$, then consider the 6 -cycle $K=x_{1} v_{1} v_{2} v_{3} x_{3} y$. By (1) the open disk bounded by $K$ includes at most one vertex of $G$. It follows that $v_{4}$ and $v_{5}$ are not inside the disk; thus either $y=x_{2}$ or $x_{2}$ is inside the disk. In either case, it follows that $x_{2}$ is 
adjacent to $x_{1}$ and $x_{3}$, a contradiction. Thus $v_{5}$ is either not $C$-admissible, or has degree at least four.

Therefore, $v_{5}$ is not a sink, and hence the final charge of $f$ is at least -3 . It follows that $v_{5}$ is not a source, which in turn implies that $v_{5}$ is $C$ admissible (because $v_{1}$ and $v_{4}$ are $C$-admissible), and hence has degree at least four. We claim that $\gamma$ is a safe pentagram. If there exists a path $P$ in $G \backslash\left\{v_{1}, v_{2}, v_{3}, v_{4}\right\}$ of length at most three with ends $x_{2}$ and $v_{5}$, then $P$ can be completed to a cycle $K$ using the path $v_{5} v_{1} v_{2} x_{2}$. By (1) we conclude that this cycle bounds an open disk that contains at most one vertex, and it follows that $x_{1}$ is adjacent to $x_{2}$, which is a contradiction. In order to complete the proof that $\gamma$ is safe it suffices to consider a path in $G \backslash\left\{v_{1}, v_{2}, v_{3}, v_{4}\right\}$ of length at most three with ends $x_{3}$ and $x_{4}$. This path can be completed via the path $x_{4} v_{4} v_{3} x_{3}$ to a cycle $K^{\prime}$. Since $v_{3}$ and $v_{4}$ have degree three, and $x_{3}$ is not adjacent to $x_{4}$, we deduce from (1) that $K^{\prime}$ is a facial cycle. Since $x_{3}$ is not adjacent to $x_{4}$ we may assume for a contradiction that $K^{\prime}$ has length six; let its vertices in order be $x_{3} v_{3} v_{4} x_{4} a b$. Then $\left(v_{4}, v_{3}, x_{3}, b, a, x_{4}\right)$ is a $C$ secure hexagram in $G$, a contradiction. This proves our claim that $\gamma$ is a safe pentagram. By symmetry the pentagram $\left(v_{4}, v_{3}, v_{2}, v_{1}, v_{5}\right)$ is also safe. We have already established that the vertices $v_{1}, v_{2}, \ldots, v_{5}, x_{1}, x_{2}, x_{3}, x_{4}$ are $C$-admissible. If $x_{i}$ has a $C$-forbidden neighbor for some $i \in\{1,2,3,4\}$, then $f$ receives one unit of charge either from that neighbor by rule (D) if $x_{i}$ has degree three, or from $x_{i}$ by rule $(\mathrm{F})$ otherwise. Since the degree of $v_{5}$ is greater than three, if $v_{5}$ has a $C$-forbidden neighbor, then it sends one unit of charge to $f$ by rule $(\mathrm{F})$. Thus at most two vertices among $v_{5}, x_{1}, x_{2}, x_{3}, x_{4}$ have a $C$-forbidden neighbor, and hence it follows that either $\gamma$, or $\left(v_{4}, v_{3}, v_{2}, v_{1}, v_{5}\right)$ is a $C$-secure pentagram, a contradiction.

Thus we have shown that $f$ has length four. Let $v_{1}, v_{2}, v_{3}, v_{4}$ be the incident vertices listed in order. Let us recall that every tetragram is safe. Since $f$ has negative charge at least $3 s-3 t$, we may assume that $v_{1}$ is a sink and $v_{3}$ is not a source. Since $v_{3}$ is not a source and $\gamma$ is not a $C$-secure tetragram, $v_{3} \in V(C)$ and $f$ is not $v_{3}$-interior. Then, (3) implies that exactly one of $v_{2} v_{3}, v_{3} v_{4}$ is an edge of $C$, and hence we may assume the latter. In particular, $v_{2} \notin V(C)$. If $v_{2}$ is a sink, then the charge of $f$ is at least -6 , otherwise it is at least -3 .

Let $v$ be the neighbor of $v_{1}$ other than $v_{2}$ and $v_{4}$. Since $v_{1}$ is a sink, $v$ is $C$-admissible. If $v$ has no $C$-forbidden neighbor, then $\gamma$ is a $C$-secure tetragram, a contradiction. Thus $v$ has a $C$-forbidden neighbor $u$. Suppose first that $u \notin V(C)$; hence $u$ is big and $f$ receives 4 units of charge from $u$ 
by rule (B). As the charge of $f$ is negative, we conclude that $v_{2}$ is a sink. Let $v^{\prime}$ be the neighbor of $v_{2}$ distinct from $v_{1}$ and $v_{3}$. Since $\gamma$ is not a $C$-secure tetragram, $v^{\prime}$ has a $C$-forbidden neighbor $u^{\prime}$. However, by rules (D) and (F), $f$ receives one unit of charge from each of $u$ and $u^{\prime}$, making its final charge nonnegative.

We conclude that every $C$-forbidden neighbor of $v$ belongs to $C$. Since rules (D) or (F) still apply, we obtain

(4) each 4-face $f$ that shares an edge with $C$ has final charge at least $-2 t$, where $t \in\{1,2\}$ is the number of sinks of $f$.

As $\gamma$ is not a $C$-secure tetragram, at least one $C$-forbidden neighbor $u$ of $v$ is adjacent to neither $v_{2}$ nor $v_{4}$. Let $C, C_{1}, C_{2}$ be the three cycles in the graph consisting of $C$ and the path $u v v_{1} v_{4}$, numbered so that $v_{3}$ belongs to $C_{2}$. We claim that $C_{2}$ has length at least seven. Note that $v_{2}$ lies in the open disk bounded by $C_{2}$; thus by (1) the cycle $C_{2}$ has length at least six. Assume that $C_{2}$ has length exactly six. By (1), the open disk it bounds contains $v_{2}$ and no other vertex of $G$. It follows that $v_{2}$ has degree three and is adjacent to $u$, which contradicts the choice of $u$.

It follows that $C_{2}$ has length at least seven, and hence $C_{1}$ has length at most five, and by the choice of $u$, it has length exactly five. By (1), $C_{1}$ bounds a face. Thus $u$ and $v_{4}$ have a common neighbor of degree two on $C$, say $z$. Let $f(\gamma)$ denote the face bounded by $C_{1}$. Let us call each tetragram for which $f(\gamma)$ is defined bad. Note that at this point, we have proved that bad tetragrams are the only faces of $G$ with negative final charge. Let $b$ be the number of bad tetragrams.

The face $f(\gamma)$ starts out with a charge of 9 , sends three units to each of $v_{1}, v, z$ by rule $(\mathrm{A})$, and receives one either from $v_{3}$ by rule (D), or from $v_{2}$ by rule $(\mathrm{F})$ for a total of +1 . Also, if there exists a tetragram $\gamma^{\prime}$ distinct from $\gamma$ such that $f(\gamma)=f\left(\gamma^{\prime}\right)$, then the final charge of $f(\gamma)$ is at least +2 . It follows that the total charge of $G$ is at least $-b$.

Since $v_{3}, v_{4}$ and $u$ have degree at least three, by (2) the total charge of $G$ is at most -3 , and so $b \geq 3$. However, since $b>1$, there must be another bad tetragram, giving at least one more vertex of $C$ of degree at least three. Therefore, the final charge of $G$ is at most -4 by (2), and hence $b \geq 4$. Let $u^{\prime}$ be the unique neighbor of $u$ in $C \backslash z$. Since $b \geq 4$ it follows by inspection that $v_{3} v_{4}$ and $u u^{\prime}$ are the only edges of $C$ that belong to a bad tetragram. We deduce that $G$ has a vertex $v^{\prime}$ of degree three with neighbors $v, v_{2}, u^{\prime}$. It follows that $\left(v, v^{\prime}, v_{2}, v_{1}\right)$ is a $C$-secure octagram, as desired. 
Proof of Theorem 4.4. Let $G$ be a triangle-free planar graph. We may assume that $G$ is actually drawn in the plane. If $G$ has a vertex of degree two or less, then it has a secure monogram, and so we may assume that $G$

has minimum degree at least three. It follows that $G$ has a facial cycle $C$ of length at most five. Let $H$ be the component of $G$ containing $C$. We may assume that $C$ bounds the outer face of $H$. Since $H$ has minimum degree at least three it follows that $V(H)-V(C) \neq \emptyset$. By Lemma $5.1 H$ has a $C$-secure multigram; but any $C$-secure multigram in $H$ is a secure multigram in $G$, as desired.

\section{Acknowledgement}

We are indebted to a referee for carefully reading the manuscript and for pointing out a couple of errors.

\section{References}

[1] K. Appel and W. Haken, Every planar map is four colorable, Part I: discharging, Illinois J. of Math. 21 (1977), 429-490.

[2] K. Appel, W. Haken and J. Koch, Every planar map is four colorable, Part II: reducibility, Illinois J. of Math. 21 (1977), 491-567.

[3] Z. Dvořák, D. Král' and R. Thomas, Coloring triangle-free graphs on surfaces, accepted to SODA'09.

[4] M. R. Garey and D. S. Johnson, Computers and intractability. A guide to the theory of NP-completeness, W. H. Freeman, San Francisco, 1979.

[5] J. Gimbel and C. Thomassen, Coloring graphs with fixed genus and girth, Trans. Amer. Math. Soc. 349 (1997), 4555-4564.

[6] H. Grötzsch, Ein Dreifarbensatz für dreikreisfreie Netze auf der Kugel, Wiss. Z. Martin-Luther-Univ. Halle-Wittenberg Math.-Natur. Reihe 8 (1959), 109-120.

[7] Ł. Kowalik, Fast 3-coloring triangle-free planar graphs, AlgorithmsESA 2004, 436-447, Lecture Notes in Comput. Sci. 3221, Springer, Berlin, 2004. 
[8] Ł. Kowalik and M. Kurowski, Oracles for bounded length shortest paths in planar graphs, ACM Trans. Algorithms 2 (2006), 335-363.

[9] B. Mohar and C. Thomassen, Graphs on Surfaces, The Johns Hopkins University Press, Baltimore and London, 2001.

[10] T. Nishizeki and N. Chiba, Planar graphs: theory and algorithms, Ann. Discr. Math. 32, North-Holland, Amsterdam, 1988.

[11] N. Robertson, D. P. Sanders, P. D. Seymour and R. Thomas, The fourcolour theorem, J. Combin. Theory Ser. B 70 (1997), 2-44.

[12] N. Robertson and P. D. Seymour, Graph Minors VII. Disjoint paths on a surface, J. Combin. Theory Ser. B 45 (1988), 212-254.

[13] W.-K. Shih and W.-L. Hsu, A new planarity test, Theoret. Comp. Sci. 223 (1999), 179-191.

[14] R. Thomas and B. Walls, Three-coloring Klein bottle graphs of girth five, J. Combin. Theory Ser. B 92 (2004), 115-135.

[15] C. Thomassen, Grötzsch's 3-color theorem and its counterparts for the torus and the projective plane, J. Combin. Theory Ser. B 62 (1994), 268-279.

[16] C. Thomassen, A short list color proof of Grötzsch's theorem, J. Combin. Theory Ser. B 88 (2003), 189-192.

[17] C. Thomassen, The chromatic number of a graph of girth 5 on a fixed surface, J. Combin. Theory Ser. B 87 (2003), 38-71.

[18] S. G. Williamson, Depth-first search and Kuratowski subgraphs, J. Assoc. Comput. Mach. 31 (1984), 681-693. 